\title{
First experience in intracranial aneurysm occlusion by balloon assisted coiling technique
}

\author{
A. Chiriac, B. Iliescu ${ }^{1}$, N. Dobrin ${ }^{1}$, I. Poeata \\ "Grigore T. Popa" University of Medicine and Pharmacy, Iasi \\ "Prof. Dr. N. Oblu" Clinic Emergency Hospital, Iasi
}

\begin{abstract}
Particular anatomical situation like wideneck aneurysms or a large neck/fundus ratio, located on an arterial bifurcation or a small artery, are still a challenge for endovascular coil occlusion. These are due to the risk of coil migration or coil protrusion into the parent vessels. In our study we describe the balloon assisted coiling technique regarding the efficacy and safety of its practice and the problems that could appear.
\end{abstract}

Key words: aneurysms, balloon remodeling technique, aneurysm coiling

\section{Introduction}

Endovascular treatment of intracranial aneurysms has evolved rapidly and become an remarkable alternative to conventional neurosurgical treatment especially due to the development and use of various detachable coils and the appropriate techniques. Even with spectacular advancement in endovascular materials and coil configuration, aneurysms with wide neck or with a large neck/fundus ratio still remain a technically challenging problem in terms of uniformly complete coil packing of the aneurysm neck.

Because of standard coil embolization technique inability to occlude wide-neck aneurysms due to the risk of coil migration or coil protrusion into the parent vessel with embolic complication, or a parent artery thrombosis, several imbunatatiri, including stent-supported coiling or balloon remodeling techniques have been incorporated into the treatment.

In 1997, Moret et al1 described a new endovascular approach of this cohort, known as balloon remodeling or balloonassisted coiling technique. The balloonassisted technique consists of temporary inflation of a nondetachable balloon across the aneurysm neck to facilitate optimal coil placement and to avoid inadvertent coil protrusion into the parent artery. The technique has been reportedly as an optimal choice in safety placement of the initial coils within wide-necked aneurysms, as a "rescue" method in cases of coil prolepses and to allow for more optimal "packing" of the aneurysm fundus and neck region (4).

In this article, we describe our first experience with selective embolization of wide-necked intracranial aneurysms, located on a small artery bifurcation, using the remodeling technique with a HyperForm balloon microcatheter.

\section{Technical procedure}

The procedure is performed with the patient under general anesthesia. The femoral access could be unilateral or bilateral. If the vascular approach is unilateral a $6 \mathrm{~F}$ vascular sheath provides access and femoral catheterization will be 
done with a $6 \mathrm{~F}$ guiding catheter equipped with a triple push-pull valve. The valve system will provide gateway for both continuous irrigation and injection of contrast (via a 2-way tap) and access for the coiling system and HyperForm balloon system.

In case of bilateral femoral access, we use a $6 \mathrm{~F}$ sheath with a $6 \mathrm{~F}$ guiding catheter for introduction of coiling and HyperForm balloon systems via a double push-pull valve, and a $5 \mathrm{~F}$ sheath with $5 \mathrm{~F}$ diagnostic catheter for angiography control. $6 \mathrm{~F}$ guiding catheter is introduced into the proximal parent artery (internal carotid artery or vertebral artery).

At first cerebral angiography is performed to evaluate morphologic features of the aneurysm and parent vessel, and to establish the optimal visual working projection for aneurysm coil occlusion. The collateral supply to the vascular territory with risk due to proposed balloon occlusion is also angiographic analysed.

A $6 \mathrm{~F}$ guiding catheter is introduced into the proximal parent artery at which the target aneurysmal lesion is located. Trough this guiding catheter a two-marker GDC microcatheter of $1.7 \mathrm{~F}$ to $2.5 \mathrm{~F}$ distal outer diameter is used to selectively microcatheterize the aneurysm. After the decision of a balloon-assisted endovascular neck bypass technique to be used, a nondetachable balloon microcatheter supported by an indwelling 0.010 - or 0.014inch microguidewire is introduced and the balloon is gently inflated within the parent artery over the neck of the aneurysm.

Sometimes in a very large neck aneurysm microcatheterisation of neck aneurysm with a HyperForm balloon can be extremely difficult. In this situation, the balloon is delivered with a microguidewire of 0.010 or 0.012 . Most commonly the microguidewire will enter inside aneurysm and not distaly into the patern artery. Thus, microguidewire is advanced into the aneurysm and easily pushed to the front along the inside wall of the aneurysm until it makes a loop inside the aneurysm following the flow direction and to go then into parent artery distal to the aneurysm. The HyperForm balloon is then advanced over the wire and placed distal to the aneurysm.
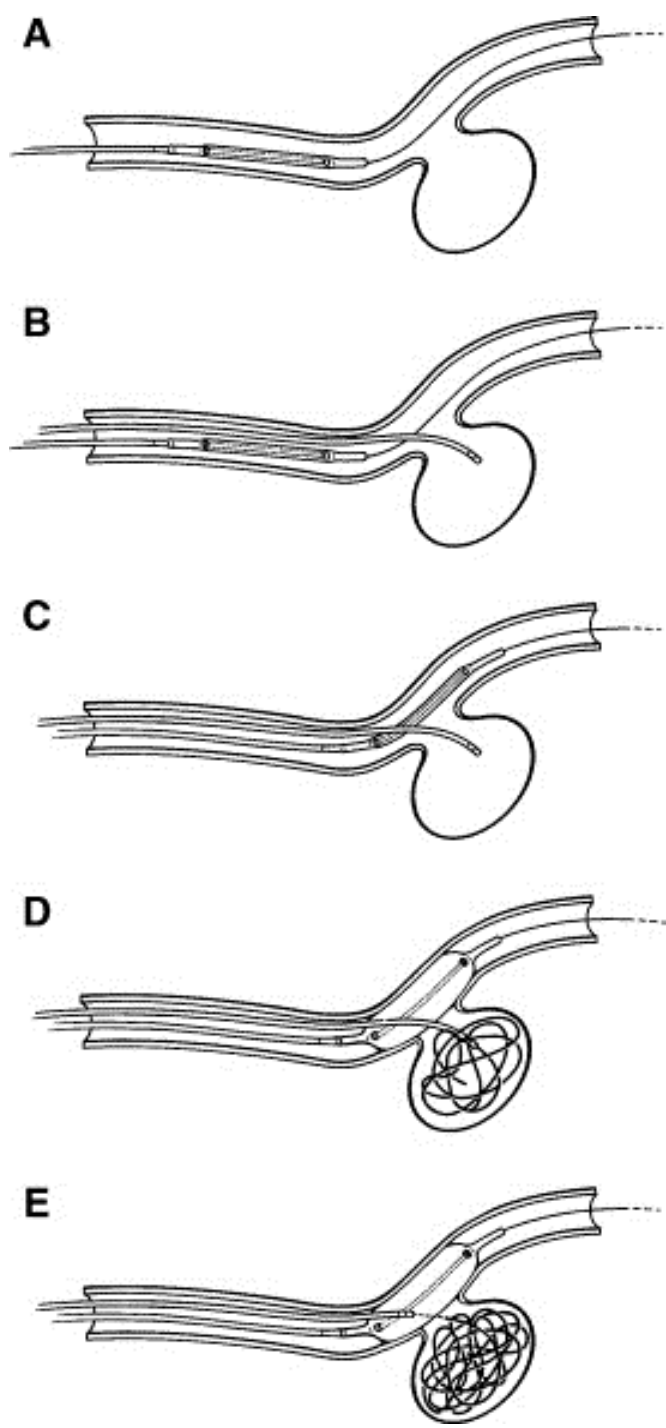

Figure 1 A - E. Steps of balloon assisted coiling technique (1) 
DOI: $10.2478 / \mathrm{v} 10282-012-0026-\mathrm{x}$

The balloon is gently inflated to stabilize it in this position. This will help to flatten the looped balloon catheter to position it bridging the neck of the aneurysm without losing the access. Then, the balloon microcatheter is pulled very slowly to remove the slackened part within the aneurysm sac. Because the balloon is inflated distally, it does not move while its loop inside the aneurysm gets straightened by gently pulling the balloon catheter. The balloon is then deflated (5).

After aneurysm neck balloon securization the initial framing coil is deployed through the intraaneurysmal microcatheter into the aneurismal sac. The balloon is immediately deflated when a stable coil configuration is considered to be reached. After balloon deflation, an interval time of 1 to 2 minutes of waiting is indicated before coil detachment to ensure that stability of coil placement could be ascertained. If no coil movement is noted, the coil is detached. The balloon is then reinflated before deployment of the next coil. The cycle is repeated until a densely packed configuration into the aneurysm is obtained. Also, the treatment is considered completed when a further balloon inflation is considered insufficient for a secure deployment of an additional coil.

The patient will received an intravenous bolus of 5000 to $7000 \mathrm{U}$ of heparin just after first coil deployment and next balloon inflation. This will be followed by intravenous infusion of 500 to $1000 \mathrm{U} / \mathrm{h}$ for the entire duration of the procedure to ensure maintenances of two times baseline of the activated coagulation time. Treatment is followed by 2 to 3 days of systemic anticoagulation with $0.3 \mathrm{U}$ fraxiparine two times per day.

\section{Case report}

A 53-year-old man known with upper gastrointestinal bleeding operated two years ago, gastroduodenal ulcers medical treated and chronic alcohol abuse was admitted to the Department of Neurosurgery in the "N. Oblu" Clinical Emergency Hospital as an emergency due to sudden headache, vertigo accompanied by by impaired balance and disturbed vision. The neurological examination revealed horizontal nystagmus and left-sided hemiparesis.

The patient was referred to an urgent non-contrast brain CT that showed a left pontine hemorrhage. Because of suspicion of a vascular pontine malformation the patient was referred also to an non-contrast brain magnetic resonance imaging examination that revealed a thin traces of subarachnoid haemorrhage filling the anterior interhemispheric and sylvian fissures and a suspicion of a left pontine cavernous malformation.

In this situation a cerebral Angio-CT was performed additionally. A middle cerebral artery bifurcation aneurysm (M1 segment) and an infundibular dilatation of the left posterior communicating artery were found.

Diagnostic procedures were complemented by a conventional cerebral angiography 12 days later. The symptom completely release at two weeks. After vascular neurosurgeon and neuroanaesthesia team consultation, the patient was proposed to an endovascular occlusion of the aneurysm due to comorbidities risks. The aneurysm was endovascular completely occluded 19 days later by a balloon assisted coiling technique. The patient's postinterventional course was uneventful. He was discharged one week after the intervention and returned to usual daily activity. 

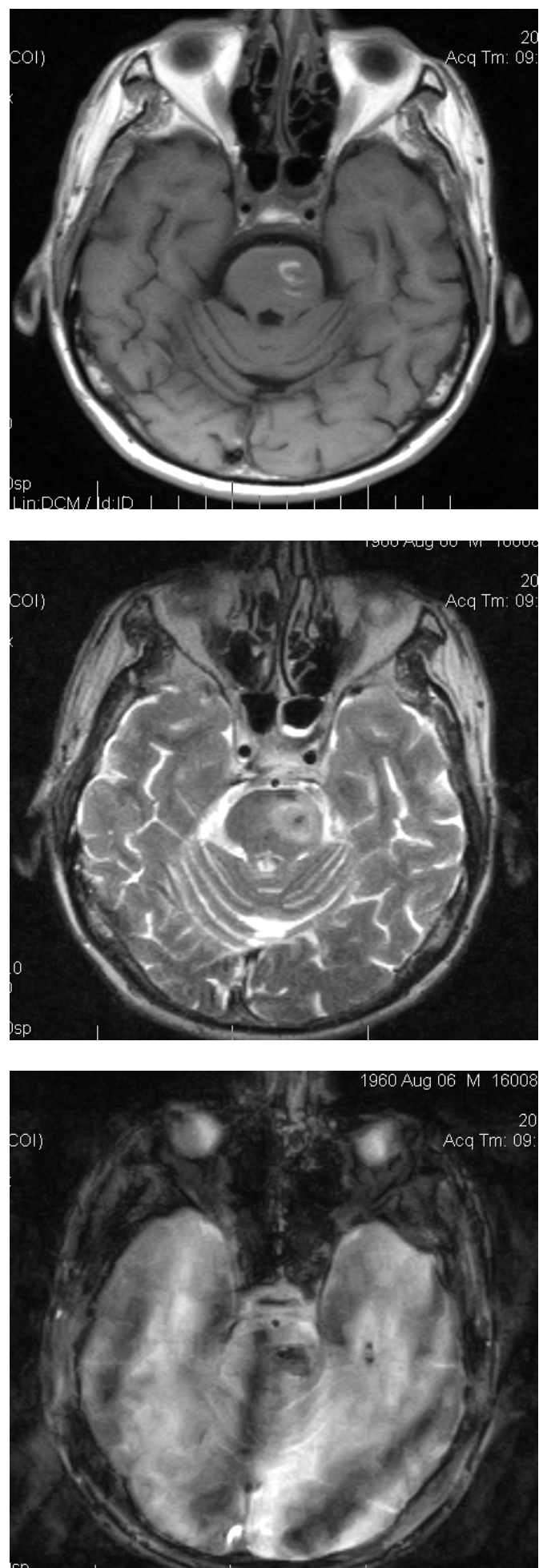

Figure 2. MRI imaging in sequences T1, T2 and $\mathrm{T} 2 \star$ showing a left pons hemorrhage due to a cavernoma

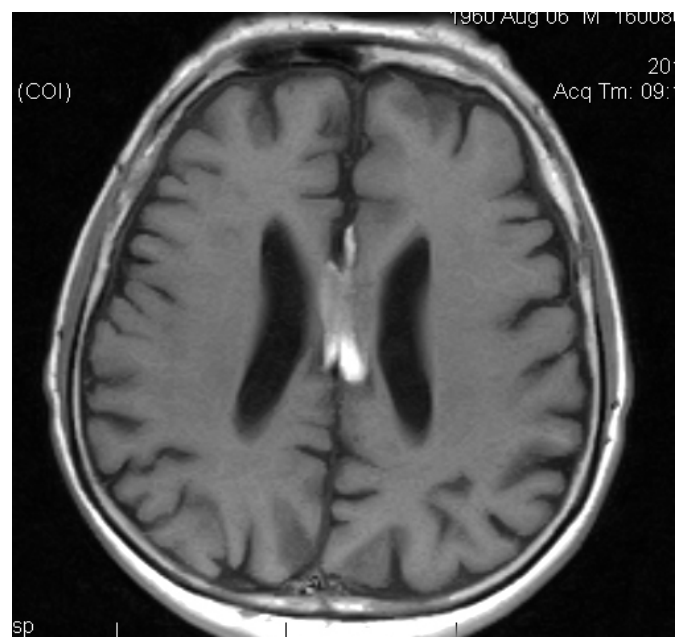

Figure 3. MRI imaging in sequences T1showing a a thin traces of interhemispheric hemorrhage
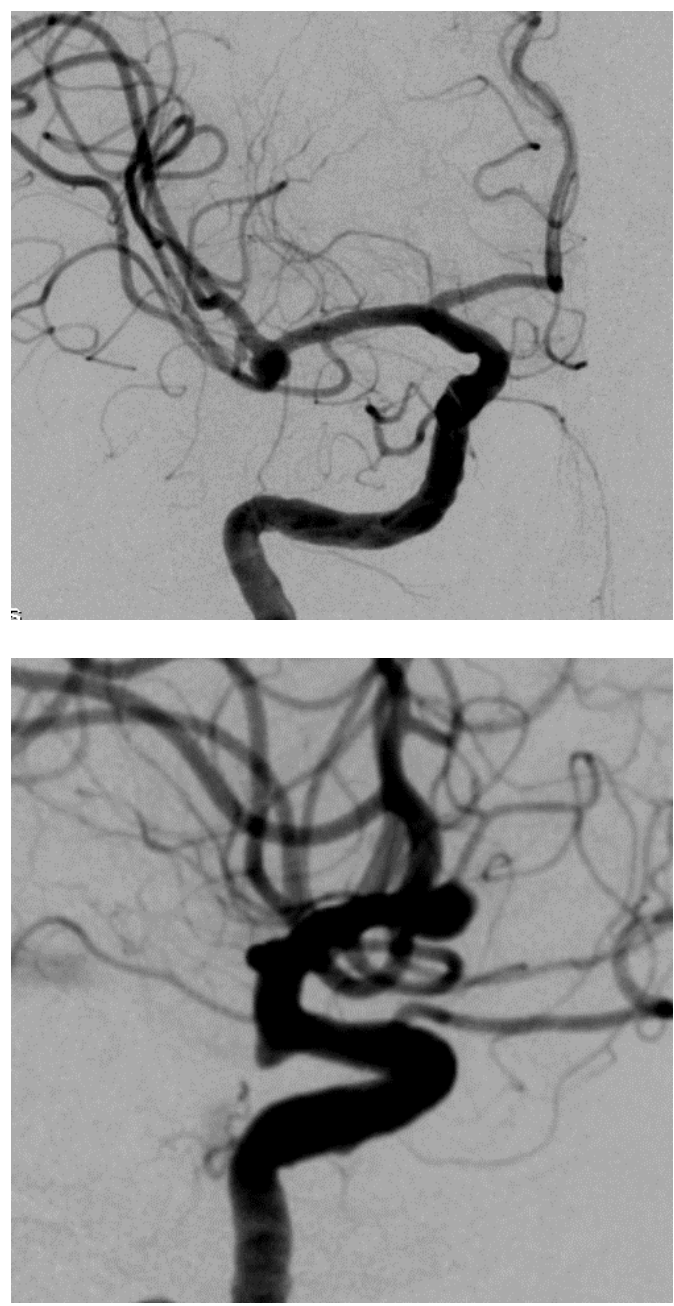

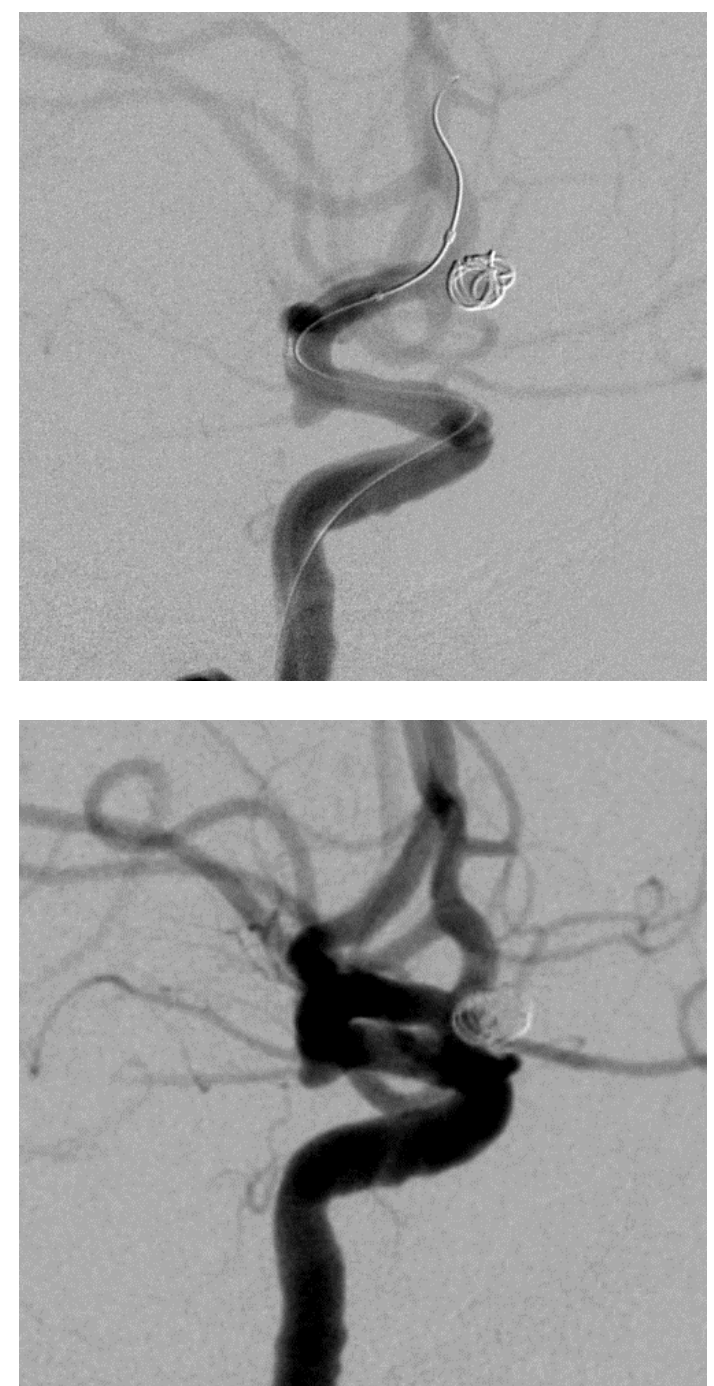

Figure 4. DSA showing a MCA aneurysm occluded by a balloon assited coiling technique

\section{Discussion}

Recently published results from the Cerebral Aneurysm Treatment Study1 suggest that balloon assisted or stent assisted coiling techniques strongly supporting efforts to achieve as complete aneurysm exclusion as possible.

The balloon-assist method has brought same additional benefits. The balloon microcatheter could serves as a reference point in the two-dimensional space of the angiographic plane due to its capability to outlines the three-dimensional course of the parent vessel during angiography. This will enable the practitioner to clearly show the aneurysm neck by an optimal orthogonal projection achievement.

Also, placing a microcatheter balloon in its uninflated or partially inflated state may be sufficient to allow optimal arrangement of coils loops that would otherwise herniated into the parent artery.

The complex bifurcation aneurysm are difficult to approached by "classic" remodelling technique with a single lowcompliance balloon due to its limitation for protection of both the neck and arterial branches. This is limited to sidewall aneurysms. These difficulties have been overcome with the introduction of a compliant latex balloon (B1; Balt, Montmorency, France) glued to the tip of a flow-dependent microcatheter (eg, Spinnaker; Target/Boston Scientific). The round shape and high compliance of such a latex balloon conforms easily to the arterial bifurcation and the flow-dependent characteristics of the microcatheter make stabilization of the balloon in the correct position sometimes difficult (6).

Some concern, however, must be tacked into consideration concerning adjunctive use of balloons. The potential problems are represented by an increased incidence of thromboembolism, even under optimized anticoagulation and aneurysmal perforation during the procedure due to balloon manipulation. The risk factors involved are the presence of a balloon in the parent vessel as promoter of stasis, thrombus formation or platelet aggregation, reaction to foreign material in the vascular system and repeated inflations and deflations of the balloon, which may lead to intimal injury. 
The incidence of thromboembolic complications during balloon-assisted embolization were reported at $8.1 \%$ in literature (7).

Another technique problem is the aneurysm perforation by microcatheter during balloon inflation or coil delivery. This may occurs due to actively deflection or pushing of microcatheter against the wall by balloon inflation, or due to advancement of the coil that may result in wall perforation because the catheter is pinned and has decreased freedom $(6,7)$.

\section{Conclusion}

The balloon assisted coiling technique was demonstrated to be a successful tool for the treatment of wide-necked or unfavorable neck/fundus ratio intracranial aneurysms, located on an arterial bifurcation or for the so-called sidewall aneurysms. The technique has improved the safety and efficacy for these patients. However, the standard balloon remodelling technique is still a challenging for some complex aneurysms located at arterial bifurcations with a small artery.

\section{References}

1. B. Ross, Gurmeet S. Dhillon : Balloon assistance as a routine adjunct to the endovascular treatment of cerebral aneurysms, Surgical Neurology 2006, 6: 593601;

2. MU Shi-qing, YANG Xin-jian, LI You-xiang, ZHANG You-ping, LÜ Ming and WU Zhong-xue : Endovascular treatment of wide-necked intracranial aneurysms using of "remodeling technique" with the HyperForm balloon, Chinese Medical Journal 2008; 121(8):725-729.

3. Sang O Youn, Jae Il Lee, Jun Kyung Ko, Tae Hong Lee, Chang Hwa Choi : Endovascular Treatment of Wide-Necked Intracranial Aneurysms Using BalloonAssisted Techniquewith HyperForm Balloon, Korean Neurosurg Soc 2010; 48 : 207-212;

4. M. Shapiro, J. Babb, T. Becske, P.K. Nelson: Safety and Efficacy of Adjunctive Balloon Remodeling during Endovascular Treatment of Intracranial Aneurysms: A Literature Review, AJNR Am J Neuroradiol 2008; 29:1777-81;

5. M. Essiga, N. Anzaloneb, S.E. Combsc, A. Dörflerd, S.-K. Leee, P. Picozzib, À. Roviraf, M. Wellerg and M. Lawh: MR Imaging of Neoplastic Central Nervous System Lesions: Review and Recommendations for Current Practice, AJNR 2012, 33: 803-817;

6. S. Baldi, C. Mounayer, M. Piotin, L. Spelle, J. Moret: Balloon-Assisted Coil Placement in Wide-Neck Bifurcation Aneurysms by Use of a New, Compliant Balloon Microcatheter, AJNR Am J Neuroradiol 2003; 24:1222-1225.

7. Sang O Youn, Jae Il Lee, Jun Kyung Ko, Tae Hong Lee, Chang Hwa Choi : Endovascular Treatment of Wide-Necked Intracranial Aneurysms Using BalloonAssisted Technique with HyperForm Balloon, J Korean Neurosurg Soc 2010; $48: 207-212$. 